\title{
Características de preservación por el método de inmersión del culmo de Guadua angustifolia Kunth (bambú), proveniente del distrito de La Florida, Cajamarca
}

\author{
Preservation characteristics by the inmersion method of \\ Guadua angustifolia Kunth (bamboo), from La Florida, Cajamarca
}

Dora A. Landauro Ponce ${ }^{1}$, Martín Araujo Flores ${ }^{2}$ y Florencio Trujillo Cuellar ${ }^{3}$

\begin{abstract}
Resumen
En el presente trabajo se han realizado pruebas para optimizar las variables del tratamiento de preservación por inmersión prolongada de secciones de Guadua angustifolia Kunth (bambú), provenientes de plantaciones del distrito de La Florida, Cajamarca. Para el desarrollo del estudio se emplearon secciones de bambú en condición seca, con tres (03) años de madurez, y se realizaron tratamientos de preservación en diferentes periodos de tiempo, utilizando una mezcla de ácido bórico y bórax (pentaborato) como preservante al $2 \%$ de concentración en solución acuosa. Asimismo se realizaron tratamientos de perforación de nudos en las secciones de bambú. Se determinó que la preservación por inmersión prolongada de secciones bambú no es efectiva sin un tratamiento de perforación de nudos, y que el compuesto químico pentaborato es efectivo para el control del ataque de termitas de la especie Cryptotermes brevis con un tiempo de inmersión de 72 horas. Previamente, se determinó la resistencia biológica del bambú al deterioro causado por los hongos de pudrición blanca Pycnoporus sanguineus y Schizophyllum conmune y por la acción de Cryptotermes brevis, en condiciones de laboratorio, usando como referencia las normas ASTM D 2017 y ASTM D 3345, respectivamente. Se obtuvo como resultado una baja resistencia biológica en ambos ensayos.
\end{abstract}

Palabras clave: Guadua angustifolia; preservación; ácido bórico; bórax; inmersión.

\begin{abstract}
The present paper shows the results of tests to optimize the preservation variables by prolonged immersion of sections of Guadua angustifolia Kunth (bamboo), originally from the plantations of La Florida District, Cajamarca. For the development of the study, bamboo sections in dry condition with three (03) years of maturity were used, and preservation treatments were realised in different periods of time, using a boric
\end{abstract}

${ }^{1}$ Consultor independiente, Perú.

${ }^{2}$ Facultad de Ciencias Forestales, Universidad Nacional Agraria La Molina, Perú. E-mail: maraujof@lamolina.edu.pe

${ }^{3}$ Facultad de Ciencias Forestales, Universidad Nacional Agraria La Molina, Perú. 
acid and borax mixture like preservative from $2 \%$ of concentration in aqueous solution. Treatments of nodal perforation were also applied in the bamboo sections. The preservation by prolonged immersion of bamboo sections is not effective without a treatment of nodal perforation, and the boric acid and borax mixture is effective by the control of the Cryptotermes brevis (dry wood termites) with 72 hours of immersion treatment. Previously, the biological resistance of bamboo was determined using cultures of Pycnoporus sanguineus and Schizophyllum commune, which cause white rotting, and Cryptotermes brevis, using the methodology of ASTM D 2017 and ASTM D 3345, respectively. A low biological resistance in both tests was obtained as a result.

Key words: Guadua angustifolia; preservation; boric acid; borax; immersion.

\section{Introducción}

El bambú es una planta de amplia distribución, común en las regiones tropicales, encontrándose principalmente en los continentes de América y Asia. Es conocida como la gramínea más grande del planeta y tiene un alto valor económico por tratarse de un recurso natural que se puede utilizar de diversas formas, desde artículos artesanales y utilitarios hasta productos industriales y componentes estructurales de puentes y edificios.

En el Perú, la superficie total de las asociaciones naturales de bambú es de aproximadamente $39978 \mathrm{~km}^{2}$, lo cual representa el 3,11\% del territorio nacional (MINAG 2008). Se encuentra en asociaciones naturales en los departamentos de Ucayali, Ica, Tumbes, Madre de Dios, San Martín, Lima, Pasco y Junín; zonas de gran potencial para las actividades de extracción, procesamiento y comercialización de la caña de bambú.

Son varias las especies identificadas en nuestro territorio, la mayoría de ellas son utilizadas en construcción, destacando entre ellas Guadua angustifolia por sus excelentes propiedades físico-mecánicas. Ascencios y Takahashi (2004) mencionan que la especie Guadua angustifolia aparentemente fue introducida en el país y se encuentra presente en 16 de los 24 departamentos del Perú. Espinoza (s.f.) indica que la superficie estimada de plantaciones, de esta especie, en el distrito de La Florida, departamento de Cajamarca es de 450 hectáreas.
El bambú, sin embargo, por ser un material de naturaleza orgánica, es susceptible al ataque de organismos biológicos causantes de deterioro. Ello representa una limitante para su uso extendido, por lo que requiere del empleo de técnicas que ayuden a prolongar su vida útil. Según Liese (2004), en Guadua angustifolia los componentes químicos de las células son: celulosa (50\%), hemicelulosa (25\%) y lignina (25\%), similares a la madera. El almidón, que se almacena como recurso energético en las células de parénquima, se encuentra entre $2 \%$ a $10 \%$. Su presencia se ve influenciada por la edad y altura del tallo, pero más decisivamente por la temporada climática. Además, no produce compuestos químicos tóxicos como el duramen de muchos árboles, por lo que su durabilidad natural es generalmente bastante baja, indicando además que el contenido de sílice (0,5-4\%) no tiene mayor impacto.

El mismo autor menciona que el bambú es susceptible al ataque de insectos y hongos de pudrición. En tallos almacenados y artesanías es muy común el deterioro causado por el escarabajo del polvo, cuyas larvas se alimentan de almidón y carbohidratos solubles presentes en el parénquima. Por otro lado, cuando los productos de bambú son sometidos a condiciones de alta humedad, el leño es susceptible principalmente al deterioro causado por hongos de pudrición blanca; y a hongos de pudrición blanda, cuando el leño está en contacto con el suelo húmedo. 
González (1986), manifiesta que la preservación tiene por objeto modificar la composición química del leño, mediante la impregnación de un preservante, haciéndolo no aceptable para los organismos biológicos. Según Liese (2004), para la preservación de bambú se debe introducir el preservante a la estructura del culmo, tan profunda y uniformemente como sea posible. Asimismo indica que la estructura anatómica presenta dificultades para un tratamiento químico eficiente, debido a que el bambú tiene más resistencia a la penetración que la madera. Además afirma que el tallo está cubierto en su capa exterior con células densamente aglomeradas, a menudo, cubierto con una capa de cera que mantiene un sello hermético que impide cualquier captación de líquidos.

El mismo autor señala que las vías principales de penetración de la solución preservante están conformadas por los vasos en el extremo cortado del tallo. Estos vasos son distribuidos de forma irregular y ocupan entre el 8 y 10 $\%$ del área total de la sección. Su orientación, fuertemente axial, se distorsiona en los nudos. Además indica que el movimiento horizontal de líquidos es necesario para que la solución preservante llegue del vaso al tejido principal circundante del parénquima y fibras, así que solo se puede distribuir por difusión y es por consiguiente un proceso lento.

Burgos (2003) y Encinas (2005) coinciden en que los tratamientos de impregnación por inmersión son los mejores para preservar culmos en estado seco, ya que en estas condiciones, la penetración de las sales se da mediante difusión simple.

Según Hidalgo (1974), para tratar el bambú por inmersión, los tallos se colocan horizontal o verticalmente dentro de un tanque con preservante por un tiempo no menor a 12 horas. Además indica que la difusión del preservante estará sujeta a factores como el espesor, la longitud de las piezas, el contenido de humedad, peso específico del leño, concentración de la solución y tiempo de impregnación. Como consecuencia de lo anterior, los tratamientos en donde se utiliza el método de inmersión, tienen una eficacia limitada y son demorados debido a la baja penetración del líquido preservante a través de la pared del culmo. Para mejorar la penetración utilizando este método, recomienda raspar la piel externa también como la pared interna, y taladrar los entrenudos para que el preservante penetre.

Morales (2006) realizó tratamientos de inmersión con sales de boro en Guadua angustifolia con el propósito de evaluar la relación entre las variables del tratamiento (concentración y tiempo de inmersión) y los parámetros de evaluación (penetración y retención). Como resultado encontró que en la mayoría de las muestras tratadas se dio una penetración total, sin embargo, se observó una zona de mayor concentración. En cuanto a la retención encontró que a concentraciones de solución de entre $3 \%$ y $9 \%$ se obtuvieron valores adecuados de protección aun para condiciones de alto riesgo de deterioro (hasta $8 \mathrm{~kg} / \mathrm{m}^{3}$ ). Finalmente, indica que el tiempo de inmersión de 3 a 5 días no afectó de forma significativa la penetración ni la retención de sales, sin embargo se observó una tendencia a tener mejores promedios de retención a medida que aumentaba el tiempo de inmersión.

Montoya (2008) evaluó la preservación de Guadua angustifolia Kunth utilizando tratamientos de inmersión, inyección y boucherie modificado, con solución de ácido bórico y bórax. Se encontró que el método de inmersión con solución de bórax y ácido bórico al $4 \%$ de concentración, trabajando con guaduas frescas y perforadas con broca de $1 / 2$ " es el de mejores resultados. El número de días de inmersión óptimo fue de 4, tiempo en el cual las guaduas dejaron de absorber la solución preservante.

Es en ese sentido, la presente investigación tiene como objetivo determinar las características de preservación de Guadua angustifolia por el método de inmersión prolongada, empleando un compuesto a base de boro como preservante. Los resultados que se generen contribuirán al conocimiento sobre las técnicas adecuadas para la preservación del bambú, 
y de esta manera, generar alternativas para un aprovechamiento racional del recurso por parte de las poblaciones rurales y un uso eficiente del mismo por parte de la industria nacional.

\section{Materiales y Métodos}

\section{Lugar de ejecución}

Las parcelas de extracción se encuentran ubicadas en las plantaciones del caserío de Limoncito, distrito de La Florida, provincia de San Miguel, departamento de Cajamarca. Los ensayos de preservación se realizaron en el Laboratorio de Preservación de la Madera de la Facultad de Ciencias Forestales de la Universidad Nacional Agraria La Molina (Lima, Perú).

\section{Materia prima}

Culmos o tallos de Guadua angustifolia Kunth, de 3 años de edad.

\section{Termites}

Se utilizaron individuos de la casta reproductora de termites de madera seca de la especie Cryptotermes brevis, en estadio de ninfa (inmaduro), recolectados a partir de madera infestada de la ciudad de Lima, los cuales fueron identificados en el Laboratorio de Preservación de la Madera - UNALM.

\section{Hongos xilófagos}

Se utilizaron cultivos aislados de los hongos Schizophyllum commune y Pycnoporus sanguineus del Laboratorio de Preservación de la Madera - UNALM.

\section{Preservante}

Se empleó un preservante inorgánico e hidrosoluble, conocido en el mercado con el nombre de "pentaborato", el cual tiene la composición que se muestra en el Cuadro 1.

\section{Reactivos}

Para determinar la presencia de boro en la madera se utilizó el reactivo de coloración de polvo turmérico, según lo indicado en AWPA - A3.

\section{Máquinas, equipos y herramientas}

- Cámaras de pudrición

- Horno de eléctrico Moore
- Incubadora Memmert

- Estufa

- Planta de impregnación de maderas a escala ECASO IE $0.31 / 11 \mathrm{P}$

- Sierra portátil de disco.

- Balanza de 0,05 g de precisión.

- Balanza analítica de 0,00005 g de precisión.

- Recipientes de inmersión.

- Varilla de metal puntiaguda.

- Martillo.

- Materiales de vidrio de uso rutinario.

\section{Metodología}

Para determinar las características de preservación de Guadua angustifolia se preservaron probetas de bambú en condición seca por el método de inmersión prolongada con el empleo de "pentaborato" como preservante, determinando el periodo de tiempo más adecuado para el tratamiento en función a los parámetros de penetración y retención del preservante. De la misma manera se evaluó el efecto de la perforación de nudos en las probetas de bambú en los resultados del tratamiento preservador.

Previamente, se determinó la resistencia biológica del bambú ante la exposición de termitas de madera seca y hongos xilófagos, en condiciones de laboratorio, y la dosis efectiva del producto preservante a la acción de termites de madera seca en probetas tratadas de bambú mediante un ensayo de valor eficaz, usando como referencia las normas ASTM D 3345 y ASTM 2017, respectivamente.

\section{Procedimiento}

\section{Selección de culmos}

Se seleccionaron aquellos culmos o tallos maduros, en plantaciones con más de 3 años de establecimiento, con un diámetro promedio de $10 \mathrm{~cm}$ aproximadamente, y de más de $15 \mathrm{~m}$ de altitud.

\begin{tabular}{|c|c|}
\hline Insumos & Proporción \\
\hline Ácido bórico $\left(\mathrm{H}_{3} \mathrm{BO}_{3}\right)$ & $50 \%$ \\
\hline Bórax $\left(\mathrm{Na}_{2} \mathrm{~B}_{4} \mathrm{O}_{7} \cdot 10 \mathrm{H}_{2} \mathrm{O}\right)$ & $50 \%$ \\
\hline
\end{tabular}

Cuadro 1. Composición del preservante "pentaborato". 


\section{Extracción}

El corte de los culmos se realizó por encima del primer entrenudo. Se hicieron cortes transversales en los culmos y se obtuvieron 8 piezas de un metro de largo para la obtención de probetas de ensayo, las cuales presentaron una cantidad similar de entrenudos. Posteriormente se envolvieron las cañas en bolsas plásticas, sellándolas herméticamente. Por último fueron embaladas en costales para así ser transportadas hacia el Laboratorio de Preservación de la Madera de la Universidad Nacional Agraria La Molina.

\section{Ensayo de durabilidad natural (prueba con hon-} gos xilófagos)

Se realizó un ensayo de durabilidad natural en concordancia con las normas NTP 251.027 y ASTM D 2017.

Las probetas de bambú fueron habilitadas con dimensiones de $5 \times 5 \mathrm{~cm}$ aproximadamente, las cuales fueron secadas a peso constante y luego sumergidas en agua destilada por un tiempo de 72 horas, para ser sometidas al ataque de hongos de pudrición blanca $(P y c-$ noporus sanguineus y Schizophyllum commune) en cámaras de pudrición durante un periodo de incubación de 3 meses a $27 \pm 2^{\circ} \mathrm{C}$.

Culminado el periodo de incubación, se secaron las probetas en estufa, determinándose el peso seco final. Con esta información se calculó la pérdida de masa de las probetas expuestas al ataque de los hongos y se determinó la resistencia de las mismas utilizando como referencia la tabla de interpretación de la norma ASTM D 2017, como se muestra en el Cuadro 2.

Ensayo de valor eficaz para el preservante de prueba

Consistió en determinar la dosis mínima efectiva de la solución preservadora contra la acción de organismos biológicos causantes de deterioro. Los organismos biológicos utilizados en el presente ensayo fueron termites de la especie Criptotermes brevis. El ensayo constó de los siguientes pasos: a. Preparación de probetas, se seleccionaron al azar 6 tallos o culmos de bambú para la extracción de 6 probetas de cada tallo. Estas se obtuvieron por cortes transversales en la zona central de los culmos, con una longitud de 5 $\mathrm{cm}$ y por corte longitudinal a través del diámetro del culmo. Finalmente, fueron sometidas a un proceso de secado hasta alcanzar el 15\% de contenido de humedad.

b. Preparación de soluciones a concentraciones gradientes, se prepararon 5 soluciones a concentraciones gradientes de preservante empleando agua destilada como solvente, siendo éstas las siguientes: a) $1 \%$; b) $2 \%$; c) $3 \%$; d) $4 \%$; y e) $5 \%$.

c. Impregnación de las probetas, por inmersión en las soluciones a concentraciones gradientes de preservante, durante un periodo de 120 horas. En cada solución preparada se trataron 6 probetas de bambú.

d. Evaluación de la impregnación, mediante la determinación de la retención calculada, utilizando la siguiente ecuación:

$$
\begin{gathered}
\text { Ret.Calc. }=\underline{(\mathrm{Pf}-\mathrm{Pi}) \times \mathrm{x} \mathrm{C} \mathrm{ff}} \\
100 \times \mathrm{Vol}
\end{gathered}
$$

Donde:

Ret. Calc: retención calculada $\left(\mathrm{kg} / \mathrm{m}^{3}\right)$

Pf: peso de la madera al finalizar el tratamiento $(\mathrm{kg})$ Pi: peso de la madera al inicio del tratamiento $(\mathrm{kg})$

Vol: volumen de madera tratada $\left(\mathrm{m}^{3}\right)$

\%C: Concentración de la solución preservadora (\%) f: factor tóxico expresado como $\mathrm{H}_{3} \mathrm{BO}_{3}$

\begin{tabular}{|c|c|c|}
\hline $\begin{array}{c}\text { Promedio de la } \\
\text { pérdida de masa } \\
\text { (\%) }\end{array}$ & $\begin{array}{c}\text { Grado de } \\
\text { resistencia a la } \\
\text { pudrición }\end{array}$ & Clases \\
\hline $0-10$ & $\begin{array}{c}\text { Altamente } \\
\text { resistente }\end{array}$ & $\mathrm{A}$ \\
\hline $11-24$ & Resistente & $\mathrm{B}$ \\
\hline $25-44$ & $\begin{array}{c}\text { Moderadamente } \\
\text { resistente }\end{array}$ & $\mathrm{C}$ \\
\hline 45 en adelante & No resistente & $\mathrm{D}$ \\
\hline
\end{tabular}

Cuadro 2. Grado de resistencia a la pudrición. Fuente: ASTM D 2017. 
Preservación por el método de inmersión del culmo de Guadua angustifolia Kunth, proveniente de Cajamarca

e. Acondicionamiento de las probetas de bambú, en estufa a $40^{\circ} \mathrm{C}$ hasta alcanzar el $15 \%$ de contenido de humedad, y luego en la incubadora a $27 \pm 2^{\circ} \mathrm{C}$ durante 72 horas.

f. Prueba de ingestión forzada, realizada en cámaras opacas, conteniendo 30 individuos de la casta reproductora en estadio de ninfas de la especie Cryptotermes brevis por cada probeta, durante 28 días de incubación a $27 \pm 2^{\circ} \mathrm{C}$. El número de repeticiones para cada tratamiento y testigo fue igual a 6 .

g. Evaluación cualitativa de la prueba, siguiendo como referencia la norma técnica ASTM D 3345, se evaluó la intensidad del daño causado por las termitas, según la siguiente escala:

10 Roeduras superficiales

9 Ataque leve

7 Ataque moderado, penetración

4 Ataque fuerte

0 Fallas

La prueba fue considerada satisfactoria cuando se alcanzó el valor de 10 de la escala empleada. Asimismo, se determinó la retención mediante un análisis de espectrofotometría de las probetas de bambú tratadas con el preservante de prueba a la concentración efectiva.

Tratamiento de preservación por inmersión prolongada

El tratamiento consistió en sumergir probetas de bambú en una solución de pentaborato a la concentración efectiva determinada, durante diferentes periodos de tiempo con el objetivo de determinar el más adecuado. Para ello, se realizaron los siguientes pasos:

a. Preparación de las probetas, secciones de culmo de un (1) metro de longitud, sometidas a un proceso de secado artificial hasta alcanzar el $15 \%$ de contenido de humedad. Se consideraron los siguientes factores para el ensayo: probetas con nudos perforados, y probetas sin nudos perforados. La perforación de nudos se realizó transversalmente, empleando una barreta de $1 / 2$ pulgada de diámetro.

b. Preparación de la solución de pentaborato, a la concentración sugerida por el ensayo de valor eficaz, empleando recipientes abiertos de 200 litros de capacidad.

\begin{tabular}{|c|c|c|c|c|}
\hline \multirow{2}{*}{$\begin{array}{c}\text { Factores de } \\
\text { tratamientos }\end{array}$} & \multicolumn{4}{|c|}{$\begin{array}{c}\text { No de repeticiones para } \\
\text { los periodos de tiempo }\end{array}$} \\
\cline { 2 - 5 } & $24 \mathrm{~h}$ & $36 \mathrm{~h}$ & $42 \mathrm{~h}$ & $108 \mathrm{~h}$ \\
\hline Bambú seco perforado & 4 & 4 & 4 & 4 \\
\hline $\begin{array}{c}\text { Bambú seco sin } \\
\text { perforar }\end{array}$ & 4 & 4 & 4 & 4 \\
\hline
\end{tabular}

Cuadro 3. Diseño experimental del ensayo de inmersión.

c. Tratamiento de inmersión, realizándose ocho (08) cargas con cuatro (04) repeticiones por carga, empleando cuatro (04) periodos de tiempo diferentes, como se muestra en el diseño experimental en el en el Cuadro 3.

d. Evaluación del tratamiento preservador, utilizando los parámetros de penetración y retención, usando como referencia la NTP 251.026. Evaluación de la madera preservada. En el caso de la penetración, esta se realizó longitudinalmente y transversalmente, seccionando las probetas por la mitad y aplicando el reactivo de coloración de polvo turmérico. $\mathrm{La}$ retención se determinó utilizando la siguiente ecuación:

$$
\operatorname{Ret}=\frac{(\mathrm{pf}-\mathrm{pi}) \times \% \mathrm{Cxf}}{\mathrm{V}^{\star} 100 \%}
$$

Donde:

Ret: retención calculada $\left(\mathrm{kg} / \mathrm{m}^{3}\right)$

pf: peso final de la probeta después del tratamiento $(\mathrm{kg})$

pi: peso inicial de la probeta antes del tratamiento $(\mathrm{kg})$

$\mathrm{V}$ : volumen de la probeta $\left(\mathrm{m}^{3}\right)$

C: concentración del preservante (\%)

f: factor de expresión en ácido bórico

También se obtuvo la retención por espectrofotometría, a partir de muestras de la zona media de las probetas ensayadas.

\section{Análisis estadístico}

Se analizaron los resultados de retención obtenidos por el tratamiento de inmersión con 


\begin{tabular}{|l|c|}
\hline \multicolumn{1}{|c|}{ Tratamientos } & Clave \\
\hline Probetas de bambú perforadas en 24 horas de inmersión & T1 \\
\hline Probetas de bambú sin perforar en 24 horas de inmersión & T2 \\
\hline Probetas de bambú perforadas en 36 horas de inmersión. & T3 \\
\hline Probetas de bambú sin perforar en 36 horas de inmersión & T4 \\
\hline Probetas de bambú perforadas en 72 horas de inmersión & T5 \\
\hline Probetas de bambú sin perforar en 72 horas de inmersión & T6 \\
\hline Probetas de bambú perforadas en 108 horas de inmersión & T7 \\
\hline Probetas de bambú sin perforar en 108 horas de inmersión & T8 \\
\hline
\end{tabular}

Cuadro 4. Clave de los tratamientos para la prueba estadística.

un diseño estadístico completamente al azar (DCA), con igual número de repeticiones; y luego, se realizó una prueba de comparación (Tukey) para conocer qué resultados son diferentes y cuales son superiores. Para el cálculo de estas pruebas se empleó un nivel de significación del $5 \%(\alpha=0,05)$, que es el más recomendado teniendo en cuenta que el número de muestras es pequeño (Calzada 1970). Para la prueba estadística se consideraron los tratamientos que se indican en el Cuadro 4.

\section{Resultados}

\section{Ensayo de durabilidad natural}

El Cuadro 5 muestra la clasificación de las probetas de Guadua angustifolia por su durabilidad natural sometidas a la acción de los hongos de pudrición blanca Schizophyllum commune y Pycnoporus sanguineus. Para este ensayo se han considerado los resultados obtenidos con el hongo xilófago que causo mayor pérdida de masa a las probetas para la clasificación del bambú por su durabilidad natural, es así que, se clasifica a estas probetas de bambú en la categoría "C": moderadamente resistente, lo que demuestra la vulnerabilidad de las muestras a la acción de estos agentes biológicos.

\section{Ensayo de valor eficaz para el preservante de prueba}

El Cuadro 6, muestra la intensidad del daño causado por termitas de madera seca en probetas de Guadua angustifolia preservadas. Asimismo, presenta la retención del compuesto

\begin{tabular}{|l|c|c|c|}
\hline \multirow{4}{*}{ Hongo xilófago } & Repetición & $\begin{array}{c}\text { Pérdida } \\
\text { de masa } \\
\text { (\%) }\end{array}$ & $\begin{array}{c}\text { Clasifi- } \\
\text { cación }\end{array}$ \\
\hline \multirow{5}{*}{$\begin{array}{l}\text { Schizophyllum } \\
\text { commune }\end{array}$} & $\mathrm{p} 1$ & 10,81 & $\mathrm{~A}$ \\
\cline { 2 - 4 } & $\mathrm{p} 2$ & 10,97 & $\mathrm{~A}$ \\
\cline { 2 - 4 } & $\mathrm{p} 3$ & 9,37 & $\mathrm{~A}$ \\
\cline { 2 - 4 } & $\mathrm{p} 4$ & 6,54 & $\mathrm{~A}$ \\
\cline { 2 - 4 } & $\mathrm{p} 5$ & 20,29 & $\mathrm{~B}$ \\
\hline \multirow{5}{*}{$\begin{array}{l}\text { Pycnoporus } \\
\text { sanguineus }\end{array}$} & $\mathrm{p} 7$ & 13,46 & $\mathrm{~B}$ \\
\cline { 2 - 4 } & $\mathrm{p} 8$ & 17,44 & $\mathrm{~B}$ \\
\cline { 2 - 4 } & $\mathrm{p} 9$ & 25,34 & $\mathrm{C}$ \\
\cline { 2 - 4 } & $\mathrm{p} 10$ & 28,15 & $\mathrm{C}$ \\
\cline { 2 - 4 } & $\mathrm{p} 11$ & 24,44 & $\mathrm{~B}$ \\
\cline { 2 - 4 } & $\mathrm{p} 12$ & 10,86 & $\mathrm{~A}$ \\
\hline \multirow{4}{*}{ Resultado } & & $\mathrm{C}$ \\
\hline
\end{tabular}

Cuadro 5. Clasificación de las probetas de Guadua angustifolia por su durabilidad natural sometidas a la acción de dos hongos de pudrición blanca. Donde: A = Altamente resistente; $\mathrm{B}=$ Resistente $\mathrm{C}=$ Modera damente resistente; $\mathrm{D}=$ No resistente, según ASTM D 2017.

\begin{tabular}{|c|c|c|c|c|c|c|}
\hline \multirow{2}{*}{$\begin{array}{c}\text { Parámetros de } \\
\text { evaluación }\end{array}$} & \multicolumn{5}{|c|}{$\begin{array}{c}\text { Concentración (\%) de las } \\
\text { Soluciones gradientes }\end{array}$} \\
\cline { 2 - 7 } & $\begin{array}{c}\text { tes- } \\
\text { tigo }\end{array}$ & $\mathbf{1}$ & $\mathbf{1 . 5}$ & $\mathbf{2}$ & $\mathbf{2 . 5}$ & $\mathbf{3}$ \\
\hline $\begin{array}{c}\text { Intensidad de } \\
\text { daño }\end{array}$ & 7 & 10 & 10 & 10 & 10 & 10 \\
\hline $\begin{array}{c}\text { Retención } \\
\text { expresado en } \\
\mathbf{k g ~ d e ~} \mathbf{H}_{\mathbf{3}} \mathbf{B O} / \mathbf{m}^{3}\end{array}$ & 0,00 & 5,28 & 5,65 & 6,21 & 6,90 & 7,09 \\
\hline
\end{tabular}

Cuadro 6. Intensidad de daños causados por Cryptotermes brevis en probetas de Guadua angustifolia preservadas con pentaborato a concentraciones gradientes. Donde: $10=$ roeduras superficiales; $9=$ ataque leve; 7 = ataque moderado, penetración; 4 = ataque fuerte; 0 = fallas. Según ASTM D 3345 .

activo determinado por espectrofotometría, observándose una relación directa entre la concentración y la retención obtenida dentro del rango ensayado.

Tomando en cuenta dichos valores, por las condiciones en las cuales las probetas han sido expuestas al preservante, para efectos de asegurar los resultados del tratamiento de inmersión 


\begin{tabular}{|c|c|c|c|c|c|c|c|c|}
\hline \multirow{2}{*}{ Repeticiones } & \multicolumn{10}{c|}{ Tratamientos } \\
\cline { 2 - 9 } & T1 & T2 & T3 & T4 & T5 & T6 & T7 & T8 \\
\hline 1 & 1,89 & 0,96 & 3,10 & 1,75 & 5,88 & 4,56 & 4,26 & 3,47 \\
2 & 2,54 & 1,51 & 3,30 & 1,93 & 5,06 & 2,21 & 5,58 & 4,71 \\
3 & 1,53 & 0,99 & 3.02 & 2,05 & 5,08 & 3,73 & 5,08 & 4,34 \\
4 & 1,68 & 1,14 & 2,69 & 2,46 & 4,32 & 1,95 & 5,39 & 4,92 \\
\hline Promedio & 1,91 & 1,15 & 3,03 & 2,05 & 5,08 & 3,11 & 5,08 & 4,36 \\
\hline
\end{tabular}

Cuadro 7. Retención calculada en probetas de Guadua angustifolia preservadas con pentaborato.

\begin{tabular}{|c|c|c|c|c|c|}
\hline \multicolumn{5}{|c|}{ Penetración y retención en probetas de Guadua angustifolia por inmersión prolongada } \\
\hline Tratamientos & $\begin{array}{c}\text { Retención de } \\
\text { pentaborato } \\
\left(\mathbf{k g} / \mathbf{m}^{3}\right)\end{array}$ & $\begin{array}{c}\text { Retención } \\
\mathbf{( k g / \mathbf { m } ^ { 3 } )} \\
\text { expresada en } \\
\text { ácido bórico }\end{array}$ & $\begin{array}{c}\text { Retención } \\
\mathbf{( k g / \mathbf { m } ^ { 3 } )} \\
\text { expresada en } \\
\text { oxido de boro }\end{array}$ & $\begin{array}{c}\text { Retención de } \\
\text { boro }\left(\mathbf{k g} / \mathbf{m}^{3}\right)\end{array}$ & Penetración \\
\hline T1 & 1,91 & 1,58 & 0,89 & 0,28 & Parcial \\
\hline T2 & 1,15 & 0,95 & 0,54 & 0,17 & Parcial \\
\hline T3 & 3,03 & 2,50 & 1,41 & 0,44 & Parcial \\
\hline T4 & 2,05 & 1,69 & 0,96 & 0,30 & Parcial \\
\hline T5 & 4,28 & 3,54 & 2,00 & 0,62 & Total \\
\hline T6 & 3,70 & 3,06 & 1,73 & 0,54 & Parcial \\
\hline T7 & 4,29 & 3,55 & 2,00 & 0,63 & Total \\
\hline T8 & 4,36 & 3,60 & 2,04 & 0,64 & Total \\
\hline
\end{tabular}

Cuadro 8. Parámetros de evaluación promedio en probetas perforadas y sin perforar de Guadua angustifolia por inmersión prolongada.

prolongada en cañas de 1 metro de longitud, se estableció una concentración de $2 \%$, ya que estas no se verán expuestas al preservante de la misma manera.

\section{Tratamiento de preservación por inmersión prolongada}

El Cuadro 7 muestra los resultados de retención calculada del ensayo de inmersión de cañas de bambú con diferentes tratamientos. En él se observa que el tiempo de inmersión influye positivamente sobre la retención calculada, a medida que este aumenta. Así mismo se observa que con el tratamiento de perforación de los nudos se obtienen resultados más altos.

En el Cuadro 8 se presentan los valores de retención promedios para cada tratamiento expresados en masa de pentaborato, ácido bórico, óxido de boro y boro molecular. Asimismo se presenta el tipo de penetración obtenido, observando que la penetración total se alcanza a las 72 horas de inmersión en cañas perforadas, y a 108 horas de inmersión en cañas sin perforación de nudos.

\section{Análisis estadístico de los resultados}

El Cuadro 9 muestra las comparaciones entre tratamientos según la metodología de análisis de Tukey para los resultados de retención de los tratamientos.

\section{Determinación de la retención por espectro- fotometría}

El Cuadro 10 muestra la retención de boro encontrado en las probetas extraídas de la zona media de las cañas de Guadua angustifolia preservadas. La retención es expresada al contenido de humedad inicial del ensayo de inmersión, que es de $15 \%$.

\section{Comparación de resultados de retención de boro}

La Figura 1 muestra la comparación de los resultados de retención de boro, en las cañas de 
bambú perforadas, calculados por diferencia de masa y por espectrofotometría. Como se puede observar, en un mismo periodo de tiempo, existen diferencias en las retenciones obtenidas con cada uno de los métodos utilizados. Se observan menores resultados de retención por el método espectrofotométrico. Asimismo, se observa una tendencia, la cual indica mejores retenciones del preservante a mayor tiempo de inmersión, sin embargo, se observa que luego

Prueba de comparación de medias de Tukey

\begin{tabular}{|c|c|c|c|}
\hline Comparaciones & dif medias & ALS(T) & Sig. \\
\hline VIII - I & 3,93 & 1,04 & $*$ \\
VIII - II & 3,17 & 1,04 & $*$ \\
VIII - III & 3,04 & 1,04 & $*$ \\
VIII - IV & 2,06 & 1,04 & $*$ \\
VIII - V & 1,97 & 1,04 & $*$ \\
VIII - VI & 0,73 & 1,04 & NS \\
VIII - VII & 0,01 & 1,04 & NS \\
\hline VII - I & 3,92 & 1,04 & $*$ \\
VII - II & 3,16 & 1,04 & $*$ \\
VII - III & 3,03 & 1,04 & $*$ \\
VII - IV & 2,05 & 1,04 & $*$ \\
VII - V & 1,96 & 1,04 & $*$ \\
VII - VI & 0,72 & 1,04 & NS \\
\hline VI - I & 3,21 & 1,04 & $*$ \\
VI - II & 2,45 & 1,04 & $*$ \\
VI - III & 2,31 & 1,04 & $*$ \\
VI - IV & 1,33 & 1,04 & $*$ \\
VI - V & 1,25 & 1,04 & $*$ \\
\hline V - I & 1,96 & 1,04 & $*$ \\
V - II & 1,20 & 1,04 & $*$ \\
V - III & 1,07 & 1,04 & $*$ \\
V - IV & 0,08 & 1,04 & NS \\
\hline IV - I & 1,88 & 1,04 & $*$ \\
IV - II & 1,12 & 1,04 & $*$ \\
IV - III & 0,98 & 1,04 & NS \\
\hline III - I & 0,89 & 1,04 & NS \\
III - II & 0,14 & 1,04 & NS \\
\hline II - I & 0,76 & 1,04 & NS \\
\hline I & & & \\
\hline & 3,04 & & $*$ \\
\hline
\end{tabular}

Cuadro 9. Comparación de promedios entre los tratamientos. de 72 horas de inmersión (3 días), en ambos casos, los resultados son constantes.

La Figura 2 muestra la comparación de los resultados de retención de boro, en las cañas de bambú sin perforar, calculados por diferencia de peso y por espectrofotometría. En ella, al igual que en el caso de las cañas perforadas, se observan diferencias en los resultados de retención obtenidos por ambos métodos y también se presenta una tendencia que indica mejores retenciones del preservante a mayor tiempo de inmersión, sin embargo, estos resultados son menores a los obtenidos en cañas perforadas. Se observa que luego de 72 horas de inmersión (3 días), los resultados arrojados por espectrofotometría son constantes.

Prueba de verificación de la eficacia del tratamiento de inmersión prolongada

Con el objetivo de verificar la eficacia del tratamiento preservador, se extrajeron probetas de la zona media de las cañas de bambú con perforación de nudos para ser sometidas a un ensayo de durabilidad con termitas de madera

\begin{tabular}{|c|c|c|}
\hline Tratamientos & $\begin{array}{c}\text { Retención } \\
\text { de boro } \\
\left(\mathrm{kg} / \mathrm{m}^{3}\right) \\
\end{array}$ & $\begin{array}{c}\text { Retención de } \\
\text { boro al } 15 \% \text { de } \\
\text { humedad }\left(\mathrm{kg} / \mathrm{m}^{3}\right)\end{array}$ \\
\hline T1 & 0,36 & 0,34 \\
\hline T2 & 0,12 & 0,13 \\
\hline T3 & 0,48 & 0,44 \\
\hline T4 & 0,17 & 0,16 \\
\hline T5 & 0,55 & 0,50 \\
\hline T6 & 0,19 & 0,18 \\
\hline T7 & 0,49 & 0,46 \\
\hline T8 & 0,14 & 0,13 \\
\hline
\end{tabular}

Cuadro 10. Cálculo de la retención por espectrofotometría.

\begin{tabular}{|c|c|c|c|c|}
\hline \multirow{2}{*}{$\begin{array}{c}\text { Intensidad } \\
\text { de daño }\end{array}$} & \multicolumn{4}{|c|}{$\begin{array}{c}\text { Tiempo de inmersión (horas) de } \\
\text { preservación }\end{array}$} \\
\cline { 2 - 5 } & $\mathbf{2 4}$ & $\mathbf{3 6}$ & $\mathbf{7 2}$ & $\mathbf{1 0 8}$ \\
\cline { 2 - 5 } & 7 & 7 & 10 & 10 \\
\hline
\end{tabular}

Cuadro 11. Intensidad del daño causado por termitas en probetas de Guadua angustifolia preservadas al $2 \%$. Donde: $10=$ roeduras superficiales; $9=$ ataque leve; 7 = ataque moderado, penetración; $4=$ ataque fuerte; 0 = fallas. Según ASTM D 3345. 


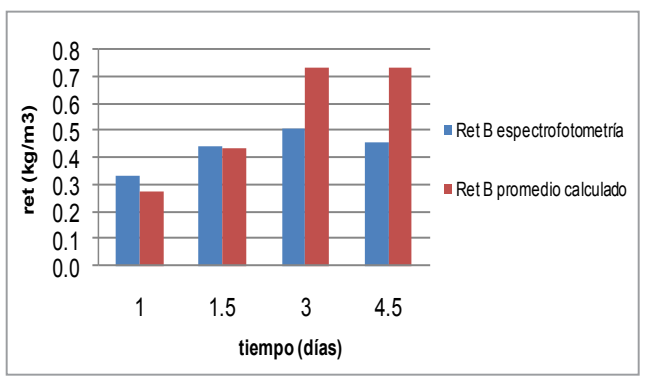

Figura 1. Retención de boro en bambú perforado.

seca. El Cuadro 11 muestra la intensidad del daño causado por las termitas de madera seca en las probetas de Guadua angustifolia preservadas. En él se observa que las probetas con un periodo de inmersión de hasta 36 horas presentan un ataque moderado, mientras que las probetas preservadas durante 72 y 108 horas no presentan daños,.

\section{Discusión}

Los resultados del ensayo de durabilidad natural concuerdan con el ensayo de valor eficaz realizado con termites como se muestra en la columna testigo del Cuadro 8, donde las probetas sufren un ataque moderado, reafirmando lo indicado por Liese (2004) que atribuye la baja resistencia del bambú a que este tiene un alto contenido de sustancias nutritivas como azúcares y almidones y no producen los componentes tóxicos que presentan la madera de duramen de muchos árboles.

A comparación de las probetas testigo, las probetas preservadas a partir de $1 \%$ de concentración no presentan daños causados por termitas, lo que significa que a estas concentraciones se alcanzan dosis tóxicas.

Se observa que no existen diferencias significativas entre los promedios de los tratamientos T6, T7 y T8, esto significa que hay evidencias estadísticas para poder afirmar que el tratamiento de cañas perforadas a 72 horas de inmersión es similar a los tratamientos de cañas perforadas y sin perforar a 108 horas, y superior al resto de tratamientos.

Las diferencias en las retenciones de boro en las cañas se deben a la distribución del preser-

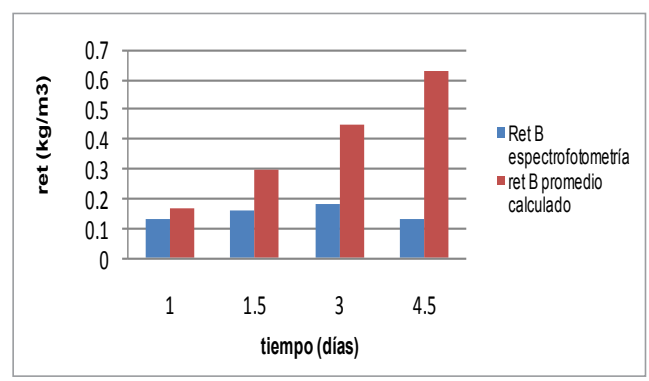

Figura 2. Retención de boro en bambú sin perforar.

vante en las piezas de bambú. Asimismo los resultados en la retención por el método espectrofotométrico se deben a la naturaleza de cómo fluyen los líquidos, lo cual hace que se presente más preservante en los extremos de las probetas que en el centro de la pieza. Los valores obtenidos por diferencias de masa serán siempre mayores, ya que con ellos se estima la retención promedio en toda la probeta.

La intensidad del daño por termitas observado significa que durante el periodo de tiempo de inmersión con una concentración de $2 \%$ se alcanzan dosis tóxicas de preservante que protegen a las probetas de bambú del ataque de termitas, con una retención efectiva $0,55 \mathrm{~kg}$ de boro $/ \mathrm{m}^{3}$ anhidro $\left(3,12 \mathrm{~kg}\right.$ de ácido bórico $\left./ \mathrm{m}^{3}\right)$.

\section{Conclusiones}

Las cañas de Guadua angustifolia son susceptibles al deterioro por acción de termitas y hongos xilófagos, siguiendo como referencia la norma técnica ASTM D 3345 y la norma ASTM D 2017.

El método de preservación por inmersión prolongada en cañas maduras (mayores de 3 años) de Guadua angustifolia, presenta mejores resultados al aplicar un proceso de secado.

La preservación por inmersión prolongada de cañas de Guadua angustifolia secas, no es efectiva sin un tratamiento de perforación de nudos.

El compuesto químico pentaborato es efectivo para el control del ataque de termitas de la especie Cryptotermes brevis. 
El tratamiento de preservación, por inmersión prolongada, es efectivo en un tiempo mínimo de 72 horas.

\section{Bibliografía}

ASTM. 2005. D 2017. Standard method of accelerated laboratory test of natural decay resistance of woods. EEUU.

ASTM. 2007. D 1413. Standard method of testing wood preservatives by laboratory soilblock cultures. EEUU.

ASTM. 2008. D 3345. Standard test method for laboratory evaluation of wood and other cellulosic materials for resistance to termites. EEUU.

AWPA 1993. A 11. Standard method for analysis of treated wood and treating solutions by atomic absorption spectroscopy. EEUU.

AWPA 2005. A 3. Standard methods for determining penetration of preservatives and fire retardants. EEUU.

Ascencios, D; Takahashi, J. 2004. Inventario de bambú en el Perú. Lima. Perú.

Burgos, A. 2003. Revisión de las técnicas de preservación del bambú. Rev. For. Lat. 33.

Calzada, J. 1970. Métodos estadísticos para la investigación. Editorial Jurídica, s.a., Tercera edición. Lima, Perú.

Encinas, O. 2005. Preservación de bambú y madera de plantaciones mediante desplazamiento de savia forzado. Revista Forestal Venezolana. Universidad de Los Andes. Mérida-Venezuela.

Espinoza, A. 2010. Aptitud de Guadua angustifolia Kunth en la elaboración de tableros aglomerados con cemento. Tesis para optar el título de ingeniero forestal. Universidad Nacional Agraria La Molina. Lima, Perú.

Giraldo, E; Sabogal, A. 1999. Una alternativa sostenible: La Guadua. Técnicas de cultivo y manejo. Colombia.

González, V. 1986. Preservación de postes de eucalipto para líneas de conducción de energía. Revista Forestal del Perú 2(1,2).
Hidalgo, O. 1974. Bambú, su cultivo y aplicaciones. Estudios Técnicos Colombianos Ltda. Cali-Colombia.

INDECOPI. 1974a. NTP 251.026. Penetración y retención de los preservadores en la madera. Perú.

INDECOPI. 1974b. NTP 251.027. Valor tóxico y permanencia de preservadores de madera en condiciones de laboratorio. Perú.

INDECOPI. 2009. NTP 251.019. Tratamientos preservadores. Definiciones y Clasificación. Perú.

Liese, W. 1998. The anatomy of bamboo culms. Technical Report. INBAR, Beijing, China.

Liese, W. 2004. Conferencia: La preservación de un tallo de bambú en relación a su estructura". Simposio Internacional Guadua 2004 Universidad Tecnológica de Pereira. Colombia.

Londoño, X. 2003. Caracterización anatómica del culmo de Guadua angustifolia Kunth (Poaceae: Bambusoideae). Bamboo, science and culture: The journal of the American Bamboo Society.

Morales, T. 2006. Evaluación y ajuste del proceso de preservación de guadua por inmersión con sales de boro. Universidad Tecnológica de Pereira- Colombia.

Montoya, J. 2008. Evaluación de métodos para la preservación de la Guadua angustifolia Kunth. Universidad Tecnológica de Pereira-Colombia.

Ministerio de Agricultura. 2008. Plan Nacional de Promoción. Fecha de consulta: 20 Agosto 2009. Disponible en: http://www.minag.gob. pe/2008/minag-lanza-plan-nacional-de-promocion.html 